\title{
MODELING OF PULSATING PERIPHERAL BIOHEAT TRANSFER USING FRACTIONAL CALCULUS AND CONSTRUCTAL THEORY
}

\author{
R. MAGIN, S. BOREGOWDA \& C. DEODHAR \\ Department of Bioengineering, University of Illinois at Chicago, Chicago, IL, USA.
}

\section{ABSTRACT}

Fractional calculus provides novel mathematical tools for modeling physical and biological processes. The bioheat equation is often used as a first order model of heat transfer in biological systems. In this paper we describe the formulation of the bioheat transfer in one dimension in terms of the fractional order differentiation with respect to time. The solution to the resulting fractional order partial differential equation reflects the interaction of the system with the dynamics of its response to the pulsed surface or volume heating and cooling. The resulting expression for the heat flux in terms of the fractional order is used to derive an expression for the depth of thermal penetration. This depth of thermal penetration, expressed as a function of time, is optimized using constructal theory, which in turn leads to the determination of the optimal time of pulsating (on-off) heating and cooling. The advantage of using the fractional heat flux expression is demonstrated by comparing the results with that of optimization of the integer heat flux expression, which yields an ideal unrealistic condition of equal time periods for optimal cooling and heating. An example from cryogenic spray cooling of a peripheral tissue region during laser surgery is used to illustrate the utility of combining the methods of fractional calculus and constructal theory. This combined approach is useful to develop an approximate solution to complex biomedical problems that involve pulsating behavior and rhythmicity.

Keywords: bioheat transfer, constructal theory, diffusion, fractals, fractional calculus, laser surgery, modeling, temperature.

\section{INTRODUCTION}

The methods of fractional calculus, reviewed recently by Magin [1], are developed as the basis for the formulation and solution of the bioheat transfer problem in peripheral tissue regions. Investigators have studied bioheat transfer using mathematical models for more than 50 years $[2,3,4]$. In these models tissue cooling (or warming) is approximated by coupling tissue perfusion to the bulk tissue temperature through Newton's law of cooling (or heating). In addition to full body models, there are numerous models in the literature that describe heat transfer mechanisms in a single organ or a portion of the body. In this regard, an analytical model developed by Keller and Seiler examines bioheat transport phenomena with heat generation (metabolism) occurring in the peripheral tissue regions. The Keller and Seiler [5] model was solved numerically using parallel computers to simulate all possible modes of bioheat transfer by Boregowda et al. [6].

Recently a number of investigators $[7,8,9]$ have applied the bioheat transfer model to periodic diffusion problems in localized tissue regions such as that which occurs in the skin when laser heating and/or cryogen cooling is applied. Fractional calculus is ideally suited to address this kind of periodic heating or cooling, but to our knowledge it has not been used in modeling bioheat transfer either at the tissue, organ or whole body level. The present study demonstrates that fractional calculus can provide a unified approach to examine the periodic heat transfer in peripheral tissue regions. For example, in an experimental study conducted by Pikkula et al. [10], cryogen spray cooling was utilized to cool the skin surface during laser skin surgery. A generalized fractional calculus approach developed by Kulish and co-workers $[11,12]$ is adopted to model the localized periodic bioheat transfer problems similar to the one posed by Pikkula and co-workers [10]. 
The one-dimensional heat flow problem can be completely solved for well-defined surface temperature or thermal flux boundary conditions by applying Laplace transforms [13,14]. The solution can also be expressed as a fractional differential equation for the semi-infinite peripheral tissue region $[11,12]$. Furthermore, the fractional differential equation can be solved to compute the heat flux at the boundary for different periodic or on-off boundary conditions that closely represent the heating and cooling of the skin surface during laser surgery.

The approach offered by the combination of fractional calculus and constructal theory models a large class of biomedical problems that involve localized pulse heating and/or cooling. The advantage of this approach is that it is not necessary to solve the entire domain and the resulting fractional heat flux expression can be used to determine the optimal heating and cooling time.

\section{GENERAL FORMULATION OF THE FRACTIONAL BIOHEAT EQUATION}

The approach used in this study is an approximation of the physical model developed in the study by Deng and Liu [7]. The region of interest is the boundary and its vicinity, and the total thickness is assumed to be large, so that rectangular coordinates in one dimension can be used for the analysis. Note that the outermost portion, the skin, is considered to be thin so that its thickness is not explicitly incorporated into the model. The localized tissue region that is represented by this approximate physical model is shown in Fig. 1.

The generalized one-dimensional bioheat transfer equation for the temperature $T(x, t)$ in the tissue developed by Pennes [15] can be written as

$$
\rho c \frac{\partial T(x, t)}{\partial t}=K \frac{\partial^{2} T(x, t)}{\partial x^{2}}+\omega_{\mathrm{b}} \rho_{\mathrm{b}} c_{\mathrm{b}}\left[T_{\mathrm{a}}-T(x, t)\right]+Q_{\mathrm{m}}+Q_{\mathrm{r}}(x, t),
$$

where $\rho, c$ and $K$ are the density, specific heat and thermal conductivity of the tissue; $\rho_{\mathrm{b}}$ and $c_{\mathrm{b}}$ are the density and specific heat of blood; $\omega_{\mathrm{b}}$ is the blood perfusion; $T_{\mathrm{a}}$ is the arterial blood temperature (assumed to be constant); $Q_{\mathrm{m}}$ is the metabolic heat generation and $Q_{\mathrm{r}}(x, t)$ is the heat generation due to spatial heating in the medium.

We assume that the problem has the following boundary conditions

$$
T\left(x, 0^{+}\right)=T_{i}(x, 0) \quad \text { Initial temperature distribution, }
$$

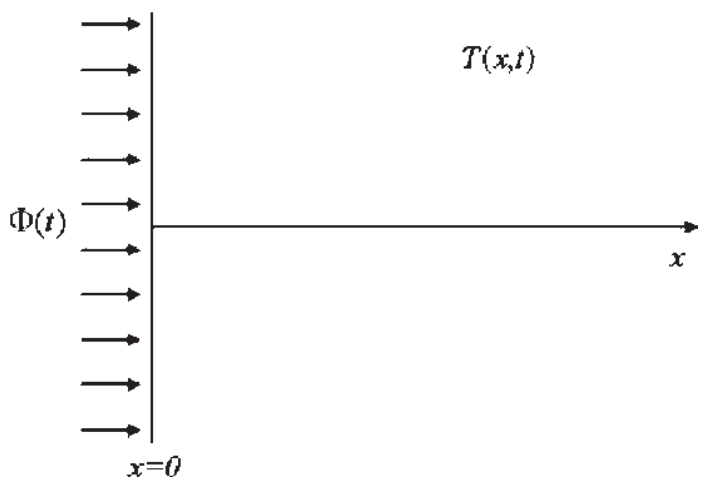

Figure 1: The assumed physical model of the localized tissue region represents the temperature in the tissue, while $\Phi(t)$ describes the surface thermal flux at $x=0$. 


$$
\begin{array}{ll}
\Phi(t)=-K \frac{\partial T(0, t)}{\partial x} & \text { Surface flux, } \\
\lim _{x \rightarrow \infty} T(x, t)=T_{\mathrm{c}} & \text { Constant core temperature. }
\end{array}
$$

If we initially assume $Q_{\mathrm{r}}$ to be zero, we can solve this problem using Liu et al.'s [9] method in terms of $\tilde{T}(x, t)=T(x, t)-T_{i}(x, 0)$, where we have subtracted the initial temperature distribution $T_{i}(x, 0)$ which is just the solution of the steady-state problem. Applying the Laplace transformation to eqn (1) for the given boundary conditions, we obtain for $h=\omega_{\mathrm{b}} \rho_{\mathrm{b}} c_{\mathrm{b}} / \rho c$ and $k=K / \rho c$

$$
\begin{array}{ll}
k \frac{\partial^{2} \tilde{t}(x, s)}{\partial x^{2}}-(s+h) \tilde{t}(x, s)=0, & \phi(s)=-K\left[\frac{\partial \tilde{t}(x, s)}{\partial x}\right]_{x=0}, \\
\tilde{T}\left(x, 0^{+}\right)=0, & \lim _{x \rightarrow \infty} \tilde{t}(x, s)=0 .
\end{array}
$$

This second order ordinary differential equation has the following solution for the specified boundary conditions

$$
\tilde{t}(x, s)=\frac{\sqrt{k} \phi(s) \mathrm{e}^{-x \sqrt{(s+h) / k}}}{K \sqrt{s+h}} .
$$

If we consider only the relationship between the flux and the temperature at the $x=0$ boundary, then the result can be written in terms of a Laplace convolution integral as

$$
\tilde{T}(0, t)=\frac{\sqrt{k}}{K} \int_{0}^{t} \Phi(t-\tau) \frac{\mathrm{e}^{-h \tau}}{\sqrt{\pi \tau}} \mathrm{d} \tau=\frac{\sqrt{k}}{K} \Phi(t) \frac{\mathrm{e}^{-h t}}{\sqrt{\pi t}},
$$

where we have used the Laplace transform pair $L^{-1}\{1 / \sqrt{s+h}\}=\mathrm{e}^{-h t} / \sqrt{\pi t}$.

Thus, if the surface flux is modeled by $\Phi(t)=\Phi_{0} u(t)$, where $u(t)$ is the unit step function, then the surface temperature will increase as

$$
\tilde{T}(0, t)=\frac{\sqrt{k}}{K} \int_{0}^{t} \frac{\Phi_{0} \mathrm{e}^{-h \tau}}{\sqrt{\pi \tau}} \mathrm{d} \tau=\frac{\sqrt{k} \Phi_{0}}{K \sqrt{h}} \frac{2}{\sqrt{\pi}} \int_{0}^{\sqrt{h t}} \mathrm{e}^{-u^{2}} \mathrm{~d} u=\frac{\sqrt{k} \Phi_{0}}{K \sqrt{h}} \operatorname{erf}(\sqrt{h t}),
$$

where $u^{2}=h \tau$ and the error function is defined by $\operatorname{erf}(x)=(2 / \sqrt{\pi}) \int_{0}^{x} \mathrm{e}^{-u^{2}} \mathrm{~d} u$.

However, the convolution integral [eqn (3)] can also be written as

$$
\tilde{T}(0, t)=\frac{\sqrt{k}}{K} \int_{0}^{t} \frac{\Phi(\tau) \mathrm{e}^{h(t-\tau)}}{\sqrt{\pi(t-\tau)}} \mathrm{d} \tau=\frac{\sqrt{k}}{K} \frac{\mathrm{e}^{-h t}}{\sqrt{\pi}} \int_{0}^{t} \frac{\Phi(\tau) \mathrm{e}^{h \tau}}{\sqrt{t-\tau}} \mathrm{d} \tau,
$$

which can be written in terms of the Riemann-Liouville fractional integral $[16,17,18,19]$ defined by

$$
{ }_{0} D_{t}^{-\alpha} F(t)=\frac{1}{\Gamma(\alpha)} \int_{0}^{t} \frac{F(\tau)}{(t-\tau)^{1-\alpha}} \mathrm{d} \tau, \quad \text { where } \Gamma(\alpha)=\int_{0}^{\infty} u^{\alpha-1} \mathrm{e}^{u} \mathrm{~d} u .
$$

Thus, eqn (4) can be simply expressed in terms of the fractional integral by

$$
\tilde{T}(0, t)=\frac{\sqrt{k}}{K} \mathrm{e}^{-h t}{ }_{0} D_{t}^{-1 / 2}\left[\mathrm{e}^{h t} \Phi(t)\right] .
$$


If we assume a step input in flux at $x=0, \Phi(t)=\Phi_{0} u(t)$, we can write

$$
\tilde{T}(0, t)=\frac{\sqrt{k}}{K} \mathrm{e}^{-h t}{ }_{0} D_{t}^{-1 / 2}\left[\Phi_{0} \mathrm{e}^{h t}\right],
$$

which, since the fractional integral is a linear operator and the fractional integral ${ }_{0} D_{t}^{-1 / 2}\left[\mathrm{e}^{h t}\right]=$ $\left(\mathrm{e}^{h t} / \sqrt{h}\right) \operatorname{erf}(\sqrt{h t})[18]$, gives the same result for the surface temperature as that obtained above by inversion of the Laplace transform.

In the case of a specified surface temperature at the surface $x=0$, a parallel analysis gives the surface flux in terms of the fractional semi-derivative of the surface temperature, which can be written

$$
\Phi(t)=\frac{K}{\sqrt{k}} \mathrm{e}^{-h t}{ }_{0} D_{t}^{1 / 2}\left[\mathrm{e}^{h t} T(0, t)\right],
$$

where the fractional derivative of order $1 / 2$ is defined [18] as

$$
{ }_{0} D_{t}^{1 / 2} F(t)=\frac{1}{\Gamma(1 / 2)} \frac{\mathrm{d}}{\mathrm{d} t} \int_{0}^{t} \frac{F(\tau)}{(t-\tau)^{1 / 2}} \mathrm{~d} \tau .
$$

This result can also be obtained using Babenko's method [19, 20].

Thus, for the case where $T(0, t)=T_{0} u(t)$, a step in the surface temperature of $T_{0}$ at $x=0$, and using the semi-derivative of $\mathrm{e}^{h t}[18]$, we obtain

$$
\Phi(t)=\frac{K T_{0}}{\sqrt{k}}\left[\frac{\mathrm{e}^{-h t}}{\sqrt{\pi t}}+\sqrt{h} \operatorname{erf}(\sqrt{h t})\right] .
$$

A graph of this result is shown in Fig. 2.

Since the relationship between flux and temperature is assumed to follow from the Fourier law for heat flux, it is valid at any point in the domain, not only at the $x=0$ surface. Therefore, for the

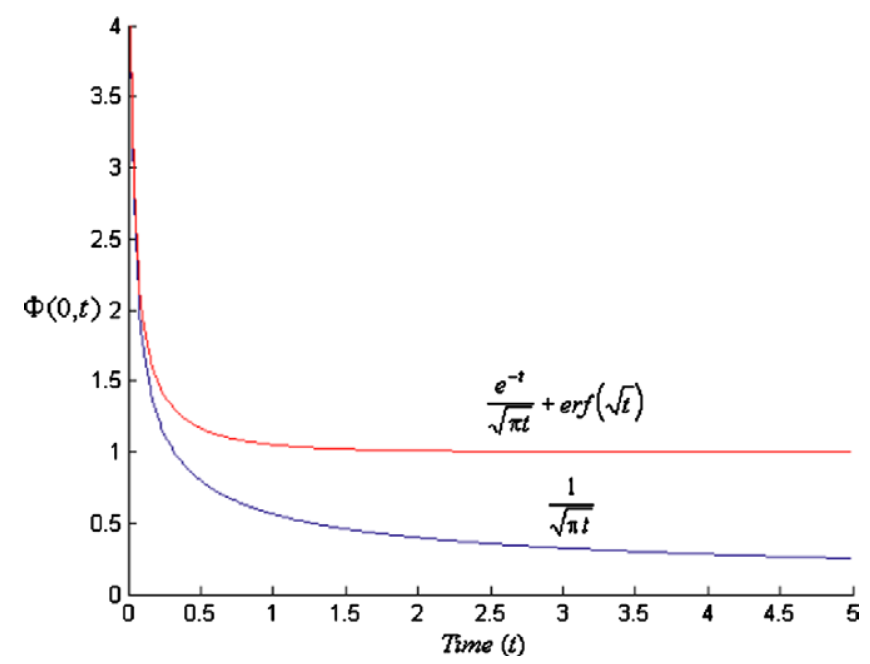

Figure 2: Graph of the flux $\Phi(0, t)$ necessary to establish a step input in the temperature $T_{0} u(t)$, assuming $A=K=k=1$. Two cases are plotted: one for the bioheat equation with $h=1$, and a second for normal diffusion without blood flow cooling, i.e. $h=0$. 
one-dimensional problem of heating with linear surface cooling, this allows us to write our fractional integral and derivative results as

$$
\Phi(x, t)=\frac{K}{\sqrt{k}} \mathrm{e}^{-h t} D_{t}^{1 / 2}\left[\mathrm{e}^{h t} \tilde{T}(x, t)\right],
$$

and

$$
\tilde{T}(x, t)=\frac{\sqrt{k}}{K} \mathrm{e}^{-h t}{ }_{0} D_{t}^{-1 / 2}\left[\mathrm{e}^{h t} \Phi(x, t)\right] .
$$

Thus, given the flux or temperature profiles at a specific location we can use this information to determine the corresponding temperature or flux. This approach could be useful in experimental situations where the half-order fractional integrals or derivatives of known functions could be used to determine the required input conditions needed for the desired temperature or flux outputs [21, 22, 23]. A few examples are listed in Table 1, which is adapted from Oldham and Spanier [18].

Note that if the initial temperature distribution $T_{i}(x, 0)$ is assumed to be uniform and constant, i.e. $T_{i}(x, 0)=T_{0}$, then $\tilde{T}(x, t)=(x, t)-T_{0}$ and the flux expression, eqn (7), becomes

$$
\Phi(x, t)=\frac{K}{\sqrt{k}} \mathrm{e}^{-h t}{ }_{0} D_{t}^{1 / 2}\left[\mathrm{e}^{h t} T(x, t)\right]-\frac{K T_{0}}{\sqrt{k}}\left[\frac{\mathrm{e}^{-h t}}{\sqrt{\pi t}}+\sqrt{h} \operatorname{erf}(\sqrt{h t})\right] .
$$

Table 1: Flux and temperature outputs for selected input functions.

\begin{tabular}{lcc}
\hline$f(t), t>0$ & $-\frac{\sqrt{k}}{K} \Phi(x, t)=\mathrm{e}^{-h t}{ }_{0} D_{t}^{1 / 2} f(t) \mathrm{e}^{h t}$ & $\frac{K}{\sqrt{k}} T(x, t)=\mathrm{e}^{-h t}{ }_{0} D_{t}^{-1 / 2} f(t) \mathrm{e}^{h t}$ \\
$A$ & $A\left[\frac{\mathrm{e}^{-h t}}{\sqrt{\pi t}}+\sqrt{h} \operatorname{erf}(\sqrt{h t})\right]$ & $A \frac{1}{\sqrt{h}} \operatorname{erf}(\sqrt{h t})$ \\
$A \mathrm{e}^{-2 h t}$ & $A\left[\frac{\mathrm{e}^{-h t}}{\sqrt{\pi t}}+\sqrt{h} \mathrm{e}^{-h t} \mathrm{erf}(\sqrt{h t})\right]$ & $A \frac{2 \mathrm{e}^{-h t}}{\sqrt{\pi h}} \mathrm{daw}(\sqrt{h t})$ \\
$A \operatorname{erf}(\sqrt{h t})$ & $A \frac{1}{\sqrt{h}}\left[1-\mathrm{e}^{-h t}\right]$ \\
$A \operatorname{erfc}(\sqrt{h t})$ & $A\left[\frac{\mathrm{e}^{-h t}}{\sqrt{\pi t}}-\sqrt{h} \operatorname{erfc}(\sqrt{h t})\right]$ & $A \frac{1}{\sqrt{h}}\left[\mathrm{e}^{-h t}-\operatorname{erfc}(\sqrt{h t})\right]$ \\
$A \operatorname{erfc}(-\sqrt{h t})$ & $A\left[\frac{\mathrm{e}^{-h t}}{\sqrt{\pi t}}+\sqrt{h} \operatorname{erfc}(-\sqrt{h t})\right]$ & 1 \\
$A / \sqrt{h t}$ & $A \sqrt{h} \frac{\sqrt{\pi}}{2} \mathrm{e}^{-h t / 2}\left[I_{1}\left(\frac{h t}{2}\right)+I_{0}\left(\frac{h t}{2}\right)\right]$ & $A \frac{\sqrt{\pi}}{\sqrt{h}} \mathrm{e}^{-h t / 2} I_{0}\left(\frac{h t}{2}\right)$ \\
$A \mathrm{e}^{-2 h t} / \sqrt{h t}$ & $A \sqrt{h} \frac{\sqrt{\pi}}{2} \mathrm{e}^{-3 h t / 2}\left[I_{1}\left(\frac{h t}{2}\right)-I_{0}\left(\frac{h t}{2}\right)\right]$ & $A \frac{\sqrt{\pi}}{\sqrt{h}} \mathrm{e}^{-3 h t / 2} I_{0}\left(\frac{h t}{2}\right)$ \\
\hline
\end{tabular}

where $\operatorname{erf}(x)=(2 / \sqrt{\pi}) \int_{0}^{x} \mathrm{e}^{-u^{2}} \mathrm{~d} u$ is the error function, $\operatorname{daw}(x)$ is Dawson's integral defined as daw $(x)=\mathrm{e}^{-x^{2}} \int_{0}^{x} \mathrm{e}^{t^{2}} \mathrm{~d} t, I_{0}(x)$ and $I_{1}(x)$ are the hyperbolic Bessel functions, and erfc $(x)$ is the complementary error function given as $\operatorname{erfc}(x)=(2 / \sqrt{\pi}) \int_{0}^{x} \mathrm{e}^{-t^{2}} \mathrm{~d} t=1-\operatorname{erf}(x)$. 
For $h=0$ this equation simplifies to

$$
\Phi(x, t)=\frac{K}{\sqrt{k}}\left[{ }_{0} D_{t}^{1 / 2} T(x, t)-\frac{T_{0}}{\sqrt{\pi t}}\right],
$$

which was previously derived by Kulish and Lage [11]. Kulish and co-workers have recently applied fractional-diffusion theory to thermoreflectance measurements of the thermal properties of thin films under pulsed laser heating. The current bioheat model under conditions of volumetric as well as surface heating extends Kulish's results, equation (10) in ref. [12], to yield

$$
\Phi(x, t)=\frac{K}{\sqrt{k}}\left\{\mathrm{e}^{-h t}{ }_{0} D_{t}^{1 / 2}\left[\mathrm{e}^{h t} \tilde{T}(x, t)\right]\right\}-\mathrm{e}^{-h t}{ }_{0} D_{t}^{1 / 2}\left[\mathrm{e}^{h t} \tilde{P}(x, t)\right]-K \frac{\partial P}{\partial x}(x, t),
$$

where $\tilde{P}(x, t)=P(x, t)-P(x, 0)$, and $P(x, t)$ represents the particular solution to the Laplace domain inhomogeneous ordinary differential equation.

This fractional calculus approach provides a simple expression for either the temperature or flux under experimental conditions often specified by laser-heating and cryogen-cooling procedures. Additional studies are needed to develop a connection between the fractional order of the operators and the material structure and properties of the tissue or substrate under study. Recent work by West et al. [24] and others [25, 26, 27] is directed toward establishing a stronger role for fractional calculus in describing dynamic phenomena in complex materials. A simple application for cryogenic skin cooling is considered in the next section.

\section{CONSTRUCTAL OPTIMIZATION OF CRYOGENIC SPRAY COOLING}

Cryogenic spray cooling is an effective technique to protect the epidermis during laser therapies. Spraying a cryogen onto the skin surface creates a time varying heat flux, effectively cooling the skin surface during and following the cryogen spurt [10,21]. In this study, we use the constructal theory developed by Bejan [28] to determine the optimal cryogenic spray cooling time. In doing so, we will consider two heat flux expressions for optimization: (i) the fractional heat flux and (ii) the integer heat flux. The objective is to compare and demonstrate the usefulness of the fractional calculus approach.

\subsection{Constructal optimization of the fractional heat flux expression}

Consider the heat flux expression for the case where the initial temperature of the skin is constant and uniform, written in terms of a fractional derivative

$$
\Phi(x, t)=\frac{K}{\sqrt{k}}\left[{ }_{0} D_{t}^{1 / 2} T(x, t)-\frac{T_{0}}{\sqrt{\pi t}}\right] .
$$

Letting $T(x, t)=a+b t$, we have $[1,18]$

$$
{ }_{0} D_{t}^{1 / 2} T(x, t)=\frac{a}{\sqrt{\pi t}}+2 b \sqrt{\frac{t}{\pi}} .
$$

Substituting eqn (11) in eqn (10), we get

$$
\Phi(x, t)=2 K b \sqrt{\frac{t}{\pi k}}+\frac{K\left(a-T_{0}\right)}{\sqrt{\pi k t}} .
$$


Rewriting eqn (12) and further simplifying, we get

$$
\Phi(x, t)=\left(\frac{2 K b}{\sqrt{\pi}}\right) \frac{t}{\delta}+\left[\frac{K\left(a-T_{0}\right)}{\sqrt{\pi}}\right] \frac{1}{\delta},
$$

where $\delta=\sqrt{k t}$ is the measure of the depth of thermal penetration due to cryogenic spray cooling or laser heating [13]. The skin layer thickness or the depth of thermal penetration $\delta(t)$ is also a measure of the effectiveness of cryogenic cooling or laser heating. In order to apply the constructal principles [28], we need to establish a relationship between the depth of thermal penetration $\delta(t)$ and time $t$. Consider the physical model from ref. [13].

The movement of the cooling front is governed by the conservation of energy in the plane $x=\delta(t)$. Consider a very wide and thin control volume that contains the cooling front and moves downward with exactly the same speed as the cooling front.

A 'stream' of cryogenic spray or liquid flow rate $\rho A(\mathrm{~d} \delta / \mathrm{d} t)$ enters the control volume from above, while another $\rho A(\mathrm{~d} \delta / \mathrm{d} t)$ stream of solid penetrates the lower skin surface (Fig. 3). The density $\rho$ refers to that of the cryogen spray liquid that contributes to the development of the whole thickness $\delta$.

Applying the first law of thermodynamics to the control volume in Fig. 3, we get

$\begin{gathered}\text { Net enthalpy flow rate out } \\ \text { of the control volume }\end{gathered}=\begin{gathered}\text { Net heat transfer rate received } \\ \text { by the same control volume }\end{gathered}$

$$
\left(\rho A \frac{\mathrm{d} \delta}{\mathrm{d} t}\right) h_{\text {cryo }}-\left(\rho A \frac{\mathrm{d} \delta}{\mathrm{d} t}\right) h_{\text {skin }}=-K A\left(\frac{\partial T}{\partial x}\right)_{x=\delta},
$$

where $A$ is the frontal area, $h_{\text {cryo }}$ is the specific enthalpy of the cryogen and $h_{\text {skin }}$ is the specific enthalpy of the skin surface. The exact analysis of the cooling phenomena requires the computation of right-hand side term of eqn (15).

From Tunnel et al. [21]

$$
-\left.K \frac{\partial T}{\partial x}\right|_{x=\delta}=\Phi(t)=h(t)\left[T_{\infty}(t)-T(0, t)\right] .
$$

But from eqn (14), we have

$$
\Phi(t)=\left(\frac{2 K b}{\sqrt{\pi}}\right) \frac{t}{\delta(t)}+\frac{K\left(a-T_{0}\right)}{\sqrt{\pi}} \frac{1}{\delta(t)} .
$$

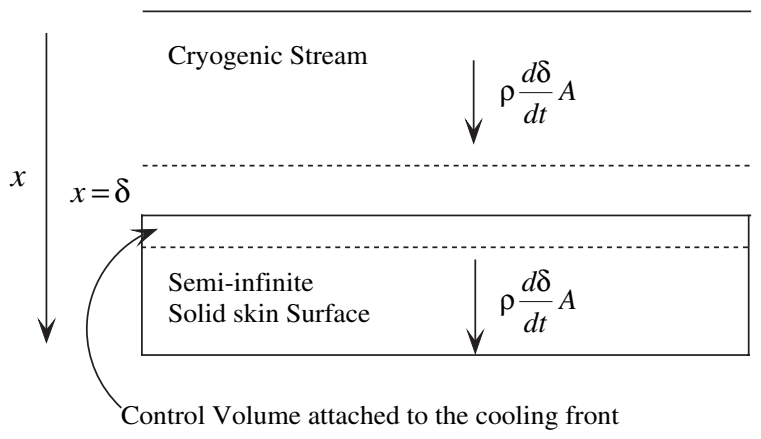

Figure 3: Cryogenic spray cooling of a semi-infinite skin surface. 
Furthermore,

$$
h_{\text {cryo,skin }}=h_{\text {cryo }}-h_{\text {skin }} .
$$

Substituting eqns (13), (16) and (17) into eqn (15), we obtain

$$
\left(\rho \frac{\mathrm{d} \delta}{\mathrm{d} t}\right) h_{\text {cryo,skin }}=\left(\frac{2 K b}{\sqrt{\pi}}\right) \frac{t}{\delta(t)}+\left[\frac{K\left(a-T_{0}\right)}{\sqrt{\pi}}\right] \frac{1}{\delta(t)} .
$$

Simplifying,

$$
\delta \frac{\mathrm{d} \delta}{\mathrm{d} t}=\left(\frac{2 K b}{\rho h_{\mathrm{cryo}, \mathrm{s}} k i n \sqrt{\pi}}\right) t+\frac{K\left(a-T_{0}\right)}{\rho A h_{\mathrm{cryo}, \mathrm{skin}} \sqrt{\pi}}
$$

for $\delta(0)=0$ (i.e. no formation of skin layer thickness).

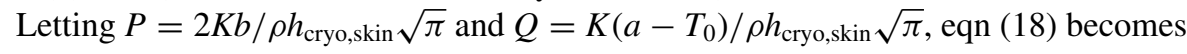

$$
\begin{aligned}
& \delta \frac{\mathrm{d} \delta}{\mathrm{d} t}=P t+Q \\
& \delta \mathrm{d} \delta=(P t+Q) \mathrm{d} t .
\end{aligned}
$$

Integrating from 0 to $t$, we obtain

$$
\delta=\left(P t^{2}+2 Q t\right)^{1 / 2} .
$$

Let us examine $\delta(t)$ (Fig. 4), the depth of penetration of the cooling front as a result of cryogenic spray cooling at the end of the freezing interval $t_{1}$, using the constructal principles from ref. [28]

$$
\delta_{1}=\left(P t_{1}+2 Q\right)^{1 / 2} t_{1}^{1 / 2}
$$

The objective is to maximize the rate of heat removal or cooling due to cryogenic spraying over the entire duration of one cycle (i.e. cryogenic cooling interval $t_{1}$ followed by the laser heating interval $t_{2}$; Fig. 5), namely

$$
\bar{\delta}=\frac{\delta_{1}}{t_{1}+t_{2}}=\frac{\left(P t_{1}+2 Q\right)^{1 / 2} t_{1}^{1 / 2}}{\left(t_{1}+t_{2}\right)} .
$$

Letting $\tau=t_{1} / t_{2}$ (non-dimensionalizing the time) and simplifying, we get

$$
\bar{\delta}=\frac{\left(P t_{2} \tau+2 Q\right)^{1 / 2}}{t_{2}^{1 / 2}} \frac{\tau^{1 / 2}}{(\tau+1)} .
$$

The only degree of freedom is the freezing time $t_{1}$ or its dimensionless counterpart, $\tau=t_{1} / t_{2}$. By solving, $\mathrm{d} \bar{\delta} / \mathrm{d} \tau=0$, we get

$$
\tau_{\mathrm{opt}}=\frac{1}{\left(1-P t_{2} / Q\right)} .
$$

Evaluating $P$ and $Q$ and simplifying further, we get

$$
t_{1, \mathrm{opt}}=\frac{1}{1-\left[2 b t_{2} /\left(a-T_{0}\right)\right]} t_{2}
$$

The optimal cryogen spray cooling time is $\left\{1 /\left[1-2 b t_{2} /\left(a-T_{0}\right)\right]\right\}$ times the laser heating interval $t_{2}$. The optimal cooling could vary with the geometry and the properties of the coolant used. 


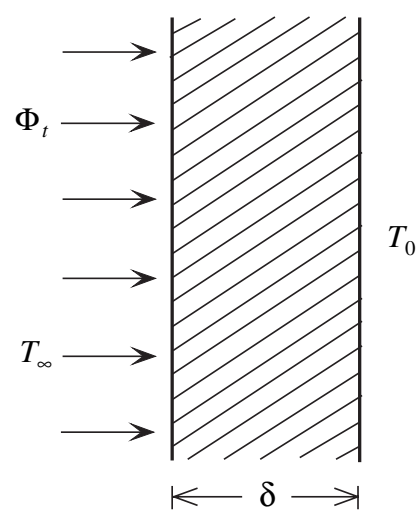

Figure 4: The depth of thermal penetration $\delta(t)$.

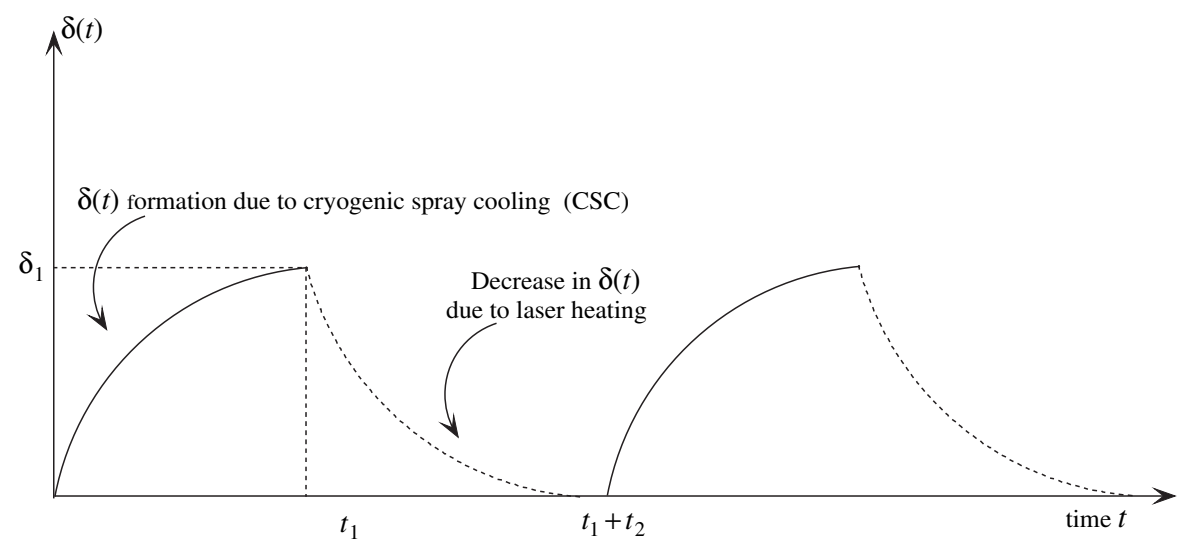

Figure 5: The intermittent production of a cryogen layer: the cooling time $t_{1}$ followed by the laser heating time $t_{2}$.

3.2 Constructal optimization of the integer heat flux expression

Consider the integer heat flux expression from heat transfer [13]

$$
\begin{aligned}
& \Phi(x, t)=\frac{-K}{\sqrt{3}} \frac{\left(T_{i}-T_{0}\right)}{(k t)^{1 / 2}} \\
& \Phi(x, t)=\frac{+K}{\sqrt{3}} \frac{\left(T_{0}-T_{i}\right)}{(k t)^{1 / 2}},
\end{aligned}
$$

where $\delta(t) \sim(k t)^{1 / 2}$

$$
\Phi(x, t)=\frac{+K\left(T_{0}-T_{i}\right)}{\sqrt{3}} \frac{1}{\delta(t)} .
$$


Rewriting eqn (18), we have

$$
\rho h \frac{\mathrm{d} \delta}{\mathrm{d} t}=\Phi(x, t)
$$

From eqns (24) and (25), we get

$$
\rho h \frac{\mathrm{d} \delta}{\mathrm{d} t}=\frac{K\left(T_{0}-T_{i}\right)}{\sqrt{3}} \frac{1}{\delta(t)} .
$$

Let us consider the initial condition: $\delta(0)=0$ (i.e. no formation of skin layer thickness or cooling front penetration).

Rewriting and solving eqn (25), we get

$$
\begin{aligned}
\delta \mathrm{d} \delta & =\frac{K\left(T_{0}-T_{i}\right)}{\sqrt{3} \rho h} \mathrm{~d} t \\
\delta \mathrm{d} \delta & =B \mathrm{~d} t, \quad \text { where } B=\frac{K\left(T_{0}-T_{i}\right)}{\sqrt{3} \rho h} \\
\frac{\delta^{2}}{2} & =B t+C \\
\delta^{2} & =2 B t+2 C \\
\delta^{2} & =2 B t+C^{\prime} \\
\delta & =\left(2 B t+C^{\prime}\right)^{1 / 2} .
\end{aligned}
$$

Applying the initial condition, we get

$$
\begin{aligned}
\delta(0) & =\left(2 \times 0+C^{\prime}\right)^{1 / 2}=0 \\
C^{\prime} & =0 \\
\delta & =\left[\frac{2 K\left(T_{0}-T_{i}\right)}{\sqrt{3} \rho h}\right]^{1 / 2} t^{1 / 2},
\end{aligned}
$$

where $B^{\prime}=\left[2 K\left(T_{0}-T_{i}\right) / \sqrt{3} \rho h\right]^{1 / 2}$

$$
\delta=B^{\prime} t^{1 / 2}
$$

Referring to Fig. 4, let us examine $\delta(t)$, the depth of thermal penetration at the end of the freezing time interval $t_{1}$, using the constructal principles [10]

$$
\delta_{1}=B^{\prime} t^{1 / 2}
$$

The objective is to maximize the rate of heat removal or cooling due to cryogenic spraying over the entire duration of one cycle, namely

$$
\bar{\delta}=\frac{\delta_{1}}{t_{1}+t_{2}}=\frac{B^{\prime} t_{1}^{1 / 2}}{\left(t_{1}+t_{2}\right)} .
$$

Letting $\tau=t_{1} / t_{2}$, we get

$$
\bar{\delta}=\frac{B^{\prime} \tau^{1 / 2}}{t_{2}^{1 / 2}(\tau+1)}=\frac{B^{\prime}}{t_{2}^{1 / 2}} \cdot \frac{\tau^{1 / 2}}{(\tau+1)}
$$


The only degree of freedom is the freezing time $t_{1}$ on its dimensionless counterpart, $\tau=t_{1} / t_{2}$. By solving, $\mathrm{d} \bar{\delta} / \mathrm{d} \tau=0$, we get

$$
\tau_{\mathrm{opt}}=1
$$

or

$$
t_{1, \mathrm{opt}}=t_{2} \text {. }
$$

This is an ideal condition that does not represent the actual pulsating heating and cooling of the peripheral skin surface. In applications such as laser skin surgery, we will need to represent the early regime heat transfer using fractional derivatives for heat flux, which in turn provides a more realistic view of the process when optimized using the constructal theory.

\section{RESULTS AND DISCUSSION}

In order to calculate the actual optimal cooling time, we tabulated the data for temperature distributions at three different skin depths from an experimental study conducted by Tunnel et al. [21]. The data is provided in Table 2 .

4.1 Constructal optimization of the fractional heat flux expression

Case (i): Depth $=20 \mu \mathrm{m}$

Performing regression analysis on the data set for depth $=20 \mu \mathrm{m}$, we get

$$
T(t)=-22.59+11.06 t,
$$

where $a=-22.59, b=11.06$ and $T_{0}=33^{\circ} \mathrm{C}$.

Table 2: Thermocouple measurements from Tunnel et al. [21] at three different depths from the skin surface.

Measured temperature $T(t)\left({ }^{\circ} \mathrm{C}\right)$

\begin{tabular}{lccc}
\cline { 2 - 4 } Time $t(\mathrm{~s})$ & Depth $=20 \mu \mathrm{m}$ & Depth $=90 \mu \mathrm{m}$ & Depth $=200 \mu \mathrm{m}$ \\
\hline 0.0 & 33.0 & 33.0 & 33.0 \\
0.2 & -50.2 & -2 & 21 \\
0.4 & -27.0 & -10 & 10 \\
0.6 & -25.0 & -2 & 10 \\
0.8 & -27.0 & 3 & 9 \\
1.0 & -26.0 & 5 & 10 \\
1.2 & -10.0 & 8 & 10 \\
1.4 & -5.0 & 12 & 14 \\
1.6 & -1.0 & 13 & 14 \\
1.8 & 0 & 15 & 15 \\
2.0 & 2 & 17 & 17 \\
2.2 & 5 & 18 & 18 \\
2.4 & 7 & 18 & 18 \\
2.6 & 9 & 19 & 19 \\
2.8 & 9 & 19 & 20 \\
3.0 & 10 & 20 & \\
\hline
\end{tabular}



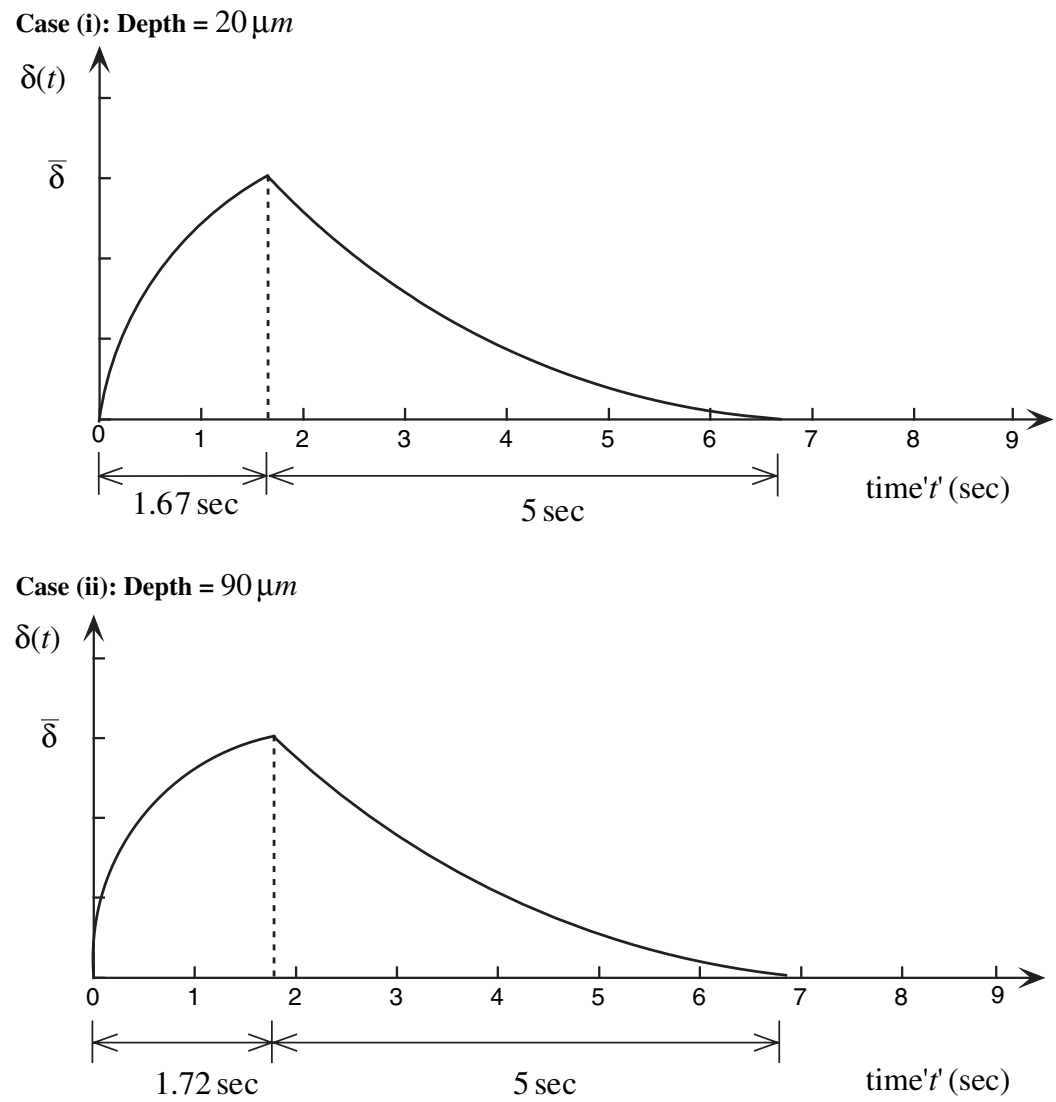

Case (iii): Depth $=200 \mu \mathrm{m}$

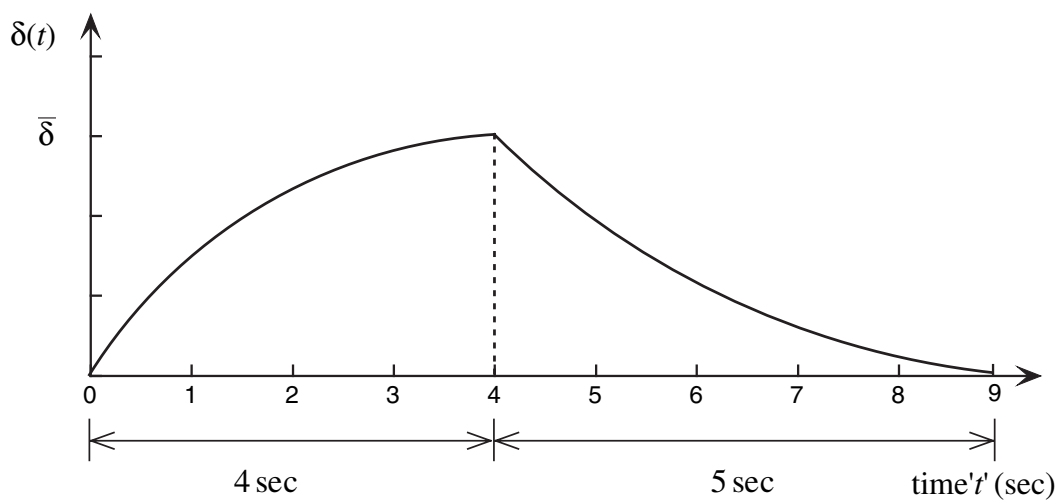

Figure 6: The optimal cooling profiles (fractional heat flux).

Thus, eqn (23) becomes

$$
t_{1, \mathrm{opt}}=\frac{t_{2}}{1+0.4 t_{2}}
$$


Case (ii): Depth $=90 \mu \mathrm{m}$

The regression equation is

$$
T(t)=3.20+5.62 t,
$$

where $a=3.20, b=5.62$ and $T_{0}=33^{\circ} \mathrm{C}$.

Thus, eqn (23) becomes

$$
t_{1, \mathrm{opt}}=\frac{t_{2}}{1+0.38 t_{2}} .
$$

Case (iii): Depth $=200 \mu \mathrm{m}$

The regression equation is

$$
T(t)=15.37+0.46 t,
$$

where $a=15.37, b=0.46$ and $T_{0}=33^{\circ} \mathrm{C}$.

Thus, eqn (23) becomes

$$
t_{1, \mathrm{opt}}=\frac{t_{2}}{1+0.05 t_{2}} .
$$

By plotting the optimal cooling time period profiles for the three different depths of penetration, we get the graphs in Fig. 6.

Using eqn (31a-c), the optimal cooling time as a function of the laser heating time is tabulated in Table 3 and graphically presented in Fig. 7.

Table 3: The distribution of optimal cryogenic spray cooling times.

\begin{tabular}{lccc} 
Laser heating & \multicolumn{3}{c}{$t_{1, \text { opt }}(\mathrm{s})$} \\
\cline { 2 - 4 } time $t_{2}(\mathrm{~s})$ & Depth $=20 \mu \mathrm{m}$ & Depth $=90 \mu \mathrm{m}$ & Depth $=200 \mu \mathrm{m}$ \\
\hline 0.0 & 0.00 & 0.00 & 0.00 \\
0.2 & 0.19 & 0.19 & 0.20 \\
0.4 & 0.34 & 0.35 & 0.39 \\
0.6 & 0.48 & 0.49 & 0.58 \\
0.8 & 0.61 & 0.61 & 0.77 \\
1.0 & 0.71 & 0.72 & 0.95 \\
1.2 & 0.81 & 0.82 & 1.13 \\
1.4 & 0.89 & 0.91 & 1.31 \\
1.6 & 0.98 & 1.00 & 1.48 \\
1.8 & 1.05 & 1.07 & 1.65 \\
2.0 & 1.11 & 1.14 & 1.82 \\
2.2 & 1.17 & 1.20 & 1.98 \\
2.4 & 1.22 & 1.26 & 2.14 \\
2.6 & 1.27 & 1.31 & 2.30 \\
2.8 & 1.32 & 1.36 & 2.46 \\
3.0 & 1.36 & 1.40 & 2.61
\end{tabular}




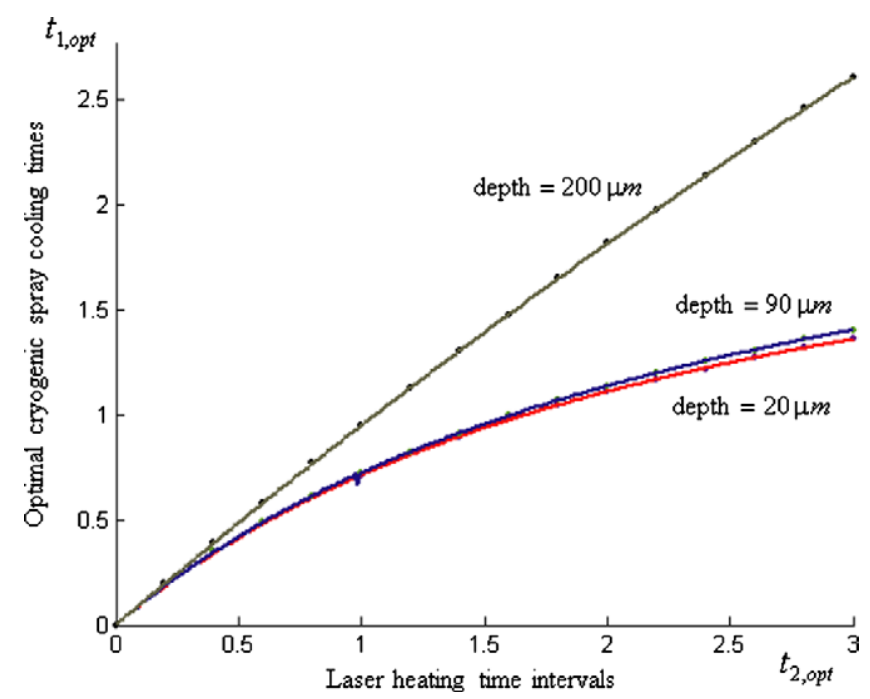

Figure 7: The variation of cryogenic spray cooling times (fractional heat flux).

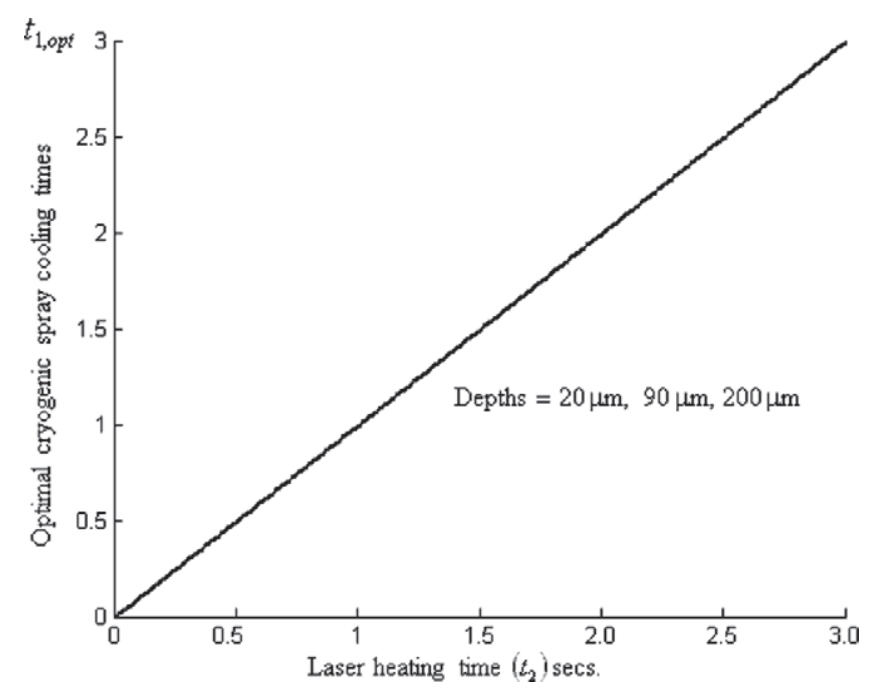

Figure 8: The variation of cryogenic cooling (integer heat flux).

4.2 Constructal optimization of the integer heat flux expression

The optimization of the integer heat flux expression results in the following expression

$$
t_{1, o p t}=t_{2} .
$$

Comparing eqn (32) with eqn (23), eqn (32) expresses the linear relationship between optimal cooling and the laser heating time periods. This is an ideal condition and not a realistic representation of the dynamic process taking place at the peripheral surface of the skin. 
Similar to the cases in Section 4.1, for illustrative purposes, let us assume that the laser heating time interval $t_{2}=5 \mathrm{~s}$. The qualitative graphical representation of the optimal cooling for all three depths is the same and is shown in Fig. 8.

\section{CONCLUSION}

The dynamics of peripheral skin thermal response to pulsed cooling or heating has been modeled as a semi-infinite heat diffusion process. As the time of pulsed cooling or heating is much shorter than the rates of blood perfusion, the approximation of a one-dimensional bioheat transfer is sufficient to investigate the pulsating nature of the cryogenic spray cooling during laser skin surgical applications. The application of fractional calculus yields an analytical expression for the heat flux in terms of fractional order derivatives. This expression is used to determine the depth of thermal penetration in the early regimes of peripheral heat transfer. The resulting depth of thermal penetration as a function of time is optimized using the constructal theory. The advantage of using the fractional heat flux expression is demonstrated by comparing the results with that of optimization of the integer heat flux expression, which yields an ideal unrealistic condition of equal time periods for optimal cooling and heating. The combined approach of using fractional calculus and constructal optimal theory offers a great potential to solve biomedical problems that involve pulsating behavior and rhythmicity. As nature affords us with rhythmicity and fine-tuned frequencies, the approach used in this paper is worthy of serious investigation by researchers interested in the field of bioengineering.

\section{REFERENCES}

[1] Magin, R.L., Fractional calculus in bioengineering. Critical Reviews in Bioengineering, 32(1-4), pp. 1-491, 2004.

[2] Wissler, E.H., Mathematical simulation of human thermal behavior using whole body models (Chapter 13). Heat Transfer in Medicine and Biology, Vol. 1, eds. A. Shitzer \& R.C. Eberhart, Plenum Press: New York, pp. 325-373, 1985.

[3] Smith, C.E., A Transient Three-Dimensional Model of the Human Thermal System, PhD Thesis, Kansas State University, Manhattan, KS, May 1991.

[4] Fu, G., A Transient, Three-Dimensional Mathematical Thermal Model for Clothed Human, $\mathrm{PhD}$ Thesis, Kansas State University, Manhattan, KS, May 1995.

[5] Keller, K.H. \& Seiler, Jr., L., An analysis of peripheral heat transfer in man. Journal of Applied Physiology, 30(5), pp. 779-786, 1971.

[6] Boregowda, S.C., Morris, J.D. \& Tiwari, S.N., Evaluation of effective thermal conductivity in peripheral tissue regions, AIAA Paper No. 98-0840, January 1998.

[7] Deng, Z-S. \& Liu, J., Analytical study on bioheat transfer problems with spatial or transient heating on skin surface or inside biological bodies. Journal of Biomechanical Engineering, 124, pp. 638-649, 2002.

[8] Anvari, B., Milner, T.E., Tanenbaum, B.S., Kimel, S., Svaasand, L.O. \& Nelson, J.S., Selective cooling of biological tissues: applications for thermally mediated therapeutic procedures. Physics in Medicine and Biology, 40, pp. 241-252, 1995.

[9] Liu, J., Zhou, Y.-X. \& Deng, Z.-S., Sinusoidal heating method to noninvasively measure tissue perfusion. IEEE Transactions on Biomedical Engineering, 49, pp. 241-252, 2003.

[10] Pikkula, P.B., Tunnell, J.W. \& Anvari, B., Methodology for characterizing heat removal mechanism in human skin during cryogen spray cooling. Annals of Biomedical Engineering, 31, pp. 493-504, 2003.

[11] Kulish, V.V. \& Lage, J.L., Fractional-diffusion solutions for transient local temperature and heat transfer. ASME Journal of Heat Transfer, 122(2), pp. 372-376, 2000. 
[12] Kulish, V.V., Lage, J.L., Komarov, P.L. \& Raad, P.E., A fractional-diffusion theory for calculating thermal properties of thin films from surface transient thermoreflectance measurements. ASME Journal of Heat Transfer, 123(6), pp. 1133-1138, 2001.

[13] Bejan, A., Heat Transfer, John Wiley \& Sons: New York, 1993.

[14] Poulikakos, D., Conduction Heat Transfer, Prentice-Hall: Englewood Cliffs, NJ, 1994.

[15] Pennes, H.H., Analysis of tissue and arterial blood temperatures in the resting human forearm. Journal of Applied Physiology, 1(2), pp. 93-122, 1948.

[16] Kochneff, E. \& Sagher, Y., Conjugate temperatures. Journal of Approximation Theory, 70, pp. 39-49, 1992.

[17] Taler, J., Theory of transient experimental techniques for surface heat transfer. International Journal of Heat and Mass Transfer, 39(17), pp. 3733-3748, 1996.

[18] Oldham, K.B. \& Spanier, J., The Fractional Calculus, Academic Press: New York, 1974.

[19] Podlubny, I.V., Fractional Differential Equations, Academic Press: San Diego, 1999.

[20] Babenko, Y.I., Heat and Mass Transfer, Khimiya: Leningrad, 1986.

[21] Tunnell, J.W., Torres, J.H. \& Anvari, B., Methodology for estimation of time-dependent surface flux due to cryogen spray cooling. Annals of Biomedical Engineering, 30, pp. 19-33, 2002.

[22] Torres, J.H., Tunnell, J.W., Pikkula, B.M. \& Anvari, B., An analysis of heat removal during cryogen spray cooling and effects of simultaneous airflow application. Lasers in Surgery and Medicine, 28, pp. 477-486, 2001.

[23] Anvari, B., Milner, B.S., Tanenbaum, B.S. \& Nelson, J.S., A comparative study of human skin thermal response to sapphire contact and cryogen spray cooling. IEEE Transactions on Biomedical Engineering, 45, pp. 934-941, 1998.

[24] West, B.J., Bologna, M. \& Grigolini, P., Physics of Fractal Operators, Springer: New York, 2003.

[25] Hilfer, R., (ed.), Applications of Fractional Calculus in Physics, World Scientific: Singapore, 2000.

[26] Carpinteri, A. \& Mainnardi, F., Fractals and Fractional Calculus in Continuum Mechanics, Springer-Verlag: New York, 1997.

[27] Bar-Yam, Y., Dynamics of Complex Systems, Perseus: Reading, 1997.

[28] Bejan, A. Shape and Structure, From Engineering to Nature, Cambridge University Press: New York, 2000. 